\title{
COMER GENTE: \\ UN ANÁLISIS DESDE LA ETNOGRAFÍA DEL HABLA
}

\author{
Mario Hernández Delgado
}

\begin{abstract}
RESUMEN
Tomando como base la metodología desarrollada por Dell Hymes para la etnografía del habla, se intenta un análisis de los componentes del acto de habla conocido en Costa Rica como comer gente. Se asume, además, que esta manifestación refleja valores y actitudes culturales específicos, los cuales se trata de explicitar de una manera introductoria.
\end{abstract}

\begin{abstract}
Based on the methodology developed by Dell Hymes for the ethnography of speaking, I intent an analysis of the components of the speech act known in Costa Rica as comer gente. Furthermore, it is assumed that this manifestation reflects specific values and specific cultural attitudes which I try to explain in an introductory way.
\end{abstract}

\section{Introducción*}

El presente trabajo consiste en el análisis de un acto social propio de nuestra cultura, al cual me referiré con el nombre de comer gente (CG). Esta es la denominación de un acto de habla tipificado culturalmente en Costa Rica, con el cual se asocia una serie de eventos, conductas e interpretaciones específicos. Para este estudio, CG interesa como acto de habla en la medida en que refleja valores y actitudes de la comunidad que lo practica. Por lo tanto, el análisis del mismo servirá como medio para develar algunos de los aspectos sicológico-sociales que implica.

Existen actos culturales -particularmente actos de habla- que por sus motivaciones o implicaciones son de alta relevancia social y a los que, sin embargo, no les ha sido asignado un nombre específico que sea parte del conocimiento cultural compartido. No obstante, el hecho de que un acto de habla (y en general cualquier acto, objeto, evento o idea) sea conocido y referido en un grupo social mediante un nombre específico (esto es, lexicalizado) es claro indicio de su significatividad para los integrantes de ese grupo. Por otro lado, CG puede 
verse como parte de un mundo semántico en el cual convergen otros actos también nominalizados, tales como chismorrear, serruchar el piso, hablar (mal) de alguien, etc.

Como miembro del mismo grupo cultural al que pertenece la manifestación de CG, estoy consciente del poder de este acto de habla como forma de manipular los sentimientos o acciones de unas personas hacia otras. Por otro lado, su influencia puede también ser refleja respecto a la misma persona que practica $\mathrm{CG}$ en forma habitual, ganándose de este modo una estereotipación social que podría afectar -generalmente en forma negativa- las relaciones de otros hacia ella.

No pretendo que sea un trabajo exhaustivo en todos los aspectos que se podrían tratar. Es, sin embargo, un primer intento de aproximarse al análisis de CG como fenómeno cultural de una comunidad de habla específica.

\section{Premisas teóricas}

\subsection{Sobre la relación lengua - cultura}

Hay dos sentidos en que se habla de la relación entre lengua y cultura. En un sentido general, consiste en una gran área de intereses que incluye las investigaciones llevadas a cabo desde corrientes con actitudes tan diferentes como la relatividad linguística y diversas formas de universalismo lingüústico, pasando por todas aquellas disciplinas surgidas del contacto entre la linguística y la sicología social, la sociología, la antropología social y la antropología cultural.

En un sentido restringido, por otro lado, es considerada como una disciplina por sí misma. No hay, sin embargo, unidad de criterios sobre su objeto propio. Rona (1974: 210), por ejemplo, afirma que las investigaciones sobre la relación entre lengua y cultura tienen como guía el estudio de las diferencias sociológicas entre dos lenguas. Para Dickeman (1982: 1189), el énfasis de esta temática ha de estar más bien en la búsqueda de generalizaciones lingüística y culturalmente universales entre esos dos factores, principalmente a través del estudio del lexicón, aunque sin olvidar el carácter sistemático del lenguaje ${ }^{1}$.

En cuanto a las investigaciones sociolinguiísticas, ambos coinciden en que consisten en la exploración de los fenómenos lingủísticos que al interior de una lengua dada están determinados por factores como sexo, edad, clase social, etc ${ }^{2}$. Así considerada, esta área, que ha llegado a ser mejor conocida como "microsociolingüística", se diferencia de la "sociolingüística macro", que incluye a aquella además de otras ramas como la etnolingủística, la linguiística antropológica, la antropología lingüística y la sociología del lenguaje. Como se puede notar, la sociolingüística, en cualquiera de sus dos presentaciones, es parte del campo de investigaciones cuyo objeto es la relación lengua-cultura siempre que se asuma la acepción más general de esta última temática.

El presente trabajo ha de valorarse en primer término desde la (macro)sociolingüística, y en particular desde la etnolinguiística, la que también se puede entender desde dos ópticas. Desde un punto de vista general, es el estudio del lenguaje en relación con su contexto cultural o, en otras palabras, el objeto de estudio de la antropología lingủística. En sentido restringido se identifica con la etnografía del habla (o de la comunicación), metodología desarrollada por Hymes cuyo objetivo es "tomar una comunidad como contexto, investigando sus hábitos de comunicación como un todo, de tal manera que cualquier uso determinado del canal y del 
código tome su lugar sólo como parte de los recursos que emplean los miembros de la comunidad" (Hymes 1974: 51).

\subsection{Sobre los recursos metodológicos}

Para este trabajo me he basado en las definiciones de las unidades sociales y en el marco general planteado por Hymes (1986). Ya hacia principios de los años sesenta, Hymes declaraba los objetivos y las tareas a que debería abocarse el entonces naciente campo de la sociolinguística, cuyo eje fundamental es la premisa de que la diversidad del habla de los individuos y de las comunidades, en apariencia asistemática, está en realidad regida por normas sociales de uso del lenguaje. Debido a que la lingüística de corte estructuralista se había interesado en estudiar los fenómenos del lenguaje casi exclusivamente "hacia adentro", es decir, como sistema relativamente cerrado, su interrelación con la vida social como un todo se había dejado de lado. La sociolingüística, por su parte, intenta ampliar el espectro de intereses de la lingüística para que incluya el uso del lenguaje y su relación con normas y valores sociales.

El primer paso en la consolidación de la sociolinguiística como disciplina científica ha consistido en establecer los conceptos y categorías que permitan contar con un aparato descriptivo coherente. Para este efecto, Hymes propone la etnografía del habla, la cual estaría llamada a ser una metodología que, compartiendo el interés de la etnografía como disciplina antropológica, ha de procurar una descripción analítica de rutinas sociales en las cuales las reglas para el uso del habla tienen un papel central ${ }^{3}$.

Existe, sin embargo, un problema más fundamental que la observación o la descripción adecuadas, y es lograr explicar la competencia comunicativa, en otras palabras: "(...) descubrir y explicar la competencia que capacita a los miembros de una comunidad para manejar e interpretar el habla (...)" (Hymes 1986: 52). Este es, por supuesto, un objetivo mucho más complejo, y no pretendo siquiera planteármelo aquí para el estudio de CG.

Según Hymes, las unidades propias de la sociolingüística deben reflejar la naturaleza social del lenguaje. El objeto matérial no será, por tanto, el lenguaje sino la comunidad de habla. Esta unidad de análisis incluye tanto la noción de comunidades sociales individuales (las cuales son de interés sociológico o antropológico) como las normas que gobiernan el uso del lenguaje al interior de tales comunidades (aspecto de interés lingüístico). En palabras de Hymes (1986: 53): "La interacción del lenguaje con la vida social es vista primero que todo como un asunto de acción humana, basada en un conocimiento, a veces consciente pero frecuentemente inconsciente, que capacita a las personas para usar el lenguaje”. Ese conocimiento, aunque requiere suponer la aptitud de los individuos para utilizar e interpretar el lenguaje de su medio, tiene que ser referido a normas de interacción lingüística. Y estas normas, a su vez, están determinadas por el conocimiento social compartido por la comunidad a que se pertenece.

\section{Objetivo}

Mi interés principal es mostrar que comer gente (CG) es un acto de habla regido por normas de producción e interpretación propias y compartidas por la comunidad linguiística de la Región Central de Costa Rica ${ }^{4}$, de la cual refleja valores y actitudes específicos. 


\section{Método de recolección, descripción y análisis de datos}

Utilizaré como base los parámetros descriptivos propuestos por Dell Hymes para la etnografía del habla, con los que se busca establecer los elementos que rigen el acontecimiento del habla en relación con el acto en estudio. Aunque para tal efecto lo ideal hubiera sido la observación directa, dada la naturaleza de CG, esto sería díficil. Por lo tanto, para la recolección de los datos he recurrido a entrevistas con informantes, de manera que la caracterización del acto está basada en sus opiniones.

Lo anterior supone variar en alguna medida el enfoque del objeto formal de estudio. En vez de describir, mediante constatación directa en el campo, bajo qué condiciones ocurre CG, el objetivo será establecer bajo qué condiciones se concibe como posible. La validez de este método se basa en considerar a los miembros de una comunidad como portadores de cultura y, por tanto, comunicativamente competentes (v. Hymes 1986: 51). En nuestro caso, se pidió a los entrevistados responder, según su experiencia personal directa, lo que conocen de otras personas de la misma comunidad lingüística o lo que creen posible según esas experiencias.

\subsection{Definición de las unidades sociales}

Las unidades sociales que analizaremos son las siguientes: primero las más englobantes, que son la comunidad linguiística y la comunidad de habla; luego, la situación de habla, el evento de habla y el acto de habla. A menos que se indique otra cosa, las definiciones han sido tomadas textualmente de Hymes (1986) o reconstruidas a partir de sus explicaciones.

Comunidad lingüística: de acuerdo con Gumperz (1974), se define como "un grupo social que puede ser monolingüe o multilingüe, unificado por la frecuencia de interacción social estructurada y separado de las áreas circunvecinas en términos de comunicación". Hymes (1986: 55) se refiere a este término como "cualquier grupo intercomunicativamente distinguible" básicamente para confrontar su alcance con el de la comunidad de habla. Para este estudio consideraré la comunidad de la Región Central de nuestro país: los entrevistados proceden de San José, Alajuela, Heredia y Cartago; además, sus actividades cotidianas se desarrollan dentro de este ámbito.

Comunidad de habla: es una "comunidad que comparte reglas para el manejo y la interpretación del habla y reglas para la interpretación de al menos una variedad linguiística"; a diferencia de la comunidad linguiística, supone una localidad común e interacción primaria. Sobre la base de que la información ofrecida por los informantes refleja también su experiencia vivida cotidianamente en un medio más amplio, me parece válido argumentar que en nuestro caso coincide con la comunidad lingüística, esto es, la misma Región Central.

Situación de habla: es una actividad integral en la cual pueden ocurrir acontecimientos verbales y no verbales. Aunque por sí misma no es gobernada por reglas de habla, provee el contexto dentro del que se desarrollan uno o varios eventos de habla. Según los informantes, el acto de CG puede tener como contexto prácticamente cualquier actividad y como momento propicio sólo se espera que haya disposición de los participantes.

Evento de habla: son "actividades, o aspectos de actividades, directamente gobernadas por reglas o normas para el uso del habla". Para CG no hay limitaciones en el evento, pudiendo coincidir con el acto a la vez que con la situación. 
Acto de habla: es la unidad mínima y constituye la producción verbal en sí. Aquí, es CG mismo, el cual consiste en un tipo de conversación de estilo espontáneo.

\subsection{Población de estudio}

Dentro de la Universidad de Costa Rica, entrevisté a un grupo de ocho personas: tres de San José, dos de Cartago, dos de Alajuela y una de Heredia. Además, me tomaré a mí mismo como informante (método introspectivo) dentro del grupo de San José; en total somos, entonces, nueve. Presentamos las siguientes características ${ }^{5}$ :

Sexo: cinco mujeres; cuatro hombres.

Edad: entre 28 y 42 años.

Estudios: todos con estudios universitarios desde un año hasta licenciatura.

Ocupación: siete realizan labores de oficina; sin embargo, cuatro de ellos han tenido otros tipos de empleos. Uno es profesor universitario, pero ha sido también bodeguero, asistente de biblioteca y agricultor. Por último, hay una estudiante recién egresada, quien también ha trabajado en oficinas.

\subsection{Notación}

i. Usaré "O" para referirme a la persona o conjunto de personas cuyas características, conductas o situaciones sean objeto de CG.

ii. La transcripción literal se indica mediante expresiones en letra cursiva a la vez que entrecomilladas. Con esto reservo el entrecomillado y la cursiva, solos, para el uso normal de resaltar algún elemento.

iii. En paréntesis cuadrados hago especificaciones o aclaraciones a las respuestas.

\section{Delimitación del objeto de estudio}

El corpus está compuesto, en concordancia con lo ya mencionado, por opiniones de los entrevistados, no por descripciones ni transcripciones de actos de CG particulares. Por otro lado, tomando en cuenta que estadísticamente priva lo general sobre lo aislado, se considerarán como más representativas las respuestas en que haya mayor coincidencia, y en estas nos concentraremos. Sin embargo, pienso que las respuestas que se desvían de la tendencia, de ningún modo se pueden catalogar como erróneas, más bien, son una importante fuente de información sobre las fronteras entre CG y otros actos emparentados. Estos no son parte de los objetivos de este estudio, aunque en algún momento haré menciones específicas a ellos.

Antes de preguntar propiamente por los componentes del acto, realicé preguntas directas sobre la existencia y la definición de CG. 


\subsection{Existencia del acto}

A la pregunta: ¿Considera usted que existe, según su experiencia personal, algo que se llame COMER GENTE?, todos los informantes respondieron afirmativamente. Además, la aserción es reforzada por dos de ellos con: "Es muy relevante en Costa Rica" y "Se da en nuestra cultura a diestra y siniestra". Con esto se verificó que efectivamente la expresión tuviera un sentido para los entrevistados.

\subsection{Definición del acto}

El pedir la definición obedeció a un doble objetivo: (a) asegurar que los informantes estuvieran considerando la expresión comer gente en la acepción metafórica que estamos tratando, en vez de su significado literal; (b) obtener una síntesis de los rasgos más característicos de CG según la propia intuición de quienes lo conocen y de la manera más espontánea y natural posible. Hay dos aspectos que interesa resaltar de las respuestas obtenidas: (a) parece haber ciertas propiedades específicas del acto de CG, de modo que a partir de ellas y de la otra información obtenida se puede abstraer la siguiente caracterización: Comer gente "es comentar sobre características o conductas de una o varias personas, en un sentido siempre negativo, y en un grupo donde necesariamente se excluye a quien o quienes son objeto de la conversación." (b) Parece haber, efectivamente, una intersección entre CG y ciertos aspectos de otros actos asociados, como "serruchar (el piso)", "hablar (mal) de alguien" y "chismorrear", a raíz de lo cual algunos informantes llegan a identificar dos o más de ellos.

\section{Exposición sistematizada de los datos}

Hymes (1986) desglosa el acto de habla en dieciséis componentes, agrupados en ocho, de acuerdo con ciertas afinidades entre algunos de ellos. Estos son: secuencia del acto (compuesta por forma y contenido del mensaje), situación del acto (separada en escenario y escena), participantes (que incluyen hablante, oyente, destinador y destinatario), propósitos (divididos en convencionales e individuales ${ }^{6}$ ), clave, instrumentales (se refieren a los canales y a las formas lingüísticas), normas (de interacción y de interpretación) y género. He tomado estos componentes como base del análisis, pero he agregado el parámetro motivos, que, aunque no es mencionado por Hymes, a mi juicio es importante para una mejor descripción de CG. Por otro lado, para las formas lingüísticas me concentro en los registros. Además, el aspecto gestual, tal como apunta Jara (1986), puede ser de suma importancia para la comprensión de ciertos actos de habla, lo cual vale también para CG; no obstante, este elemento lo examino dentro de la forma del acto, aunque desde otra perspectiva se podría separar.

Es menester tener claro que un acto cultural es una totalidad, disgregable sólo para efectos de análisis, y que la secuencia en que se expongan los diversos componentes es, en principio, arbitraria ${ }^{7}$. Por eso mismo, me he tomado la libertad de alterar el orden en que Hymes diserta sobre ellos.

Antes de comenzar el análisis de CG, es conveniente hacer la siguiente acotación: entre la escena, los propósitos convencionales, los propósitos individuales y los motivos (y también, aunque menos directamente evidente, en la clave) parece haber cierto paralelismo en cuanto a la 
existencia de dos tipos básicos de respuestas. Debido a eso, para clasificar las respuestas sobre los componentes citados he recurrido a un criterio análogo en todos ellos. Incluyo dentro de la que llamo clase A aquellas menciones que hacen referencia a la diversión o el entretenimiento como posibles determinantes de los componentes del acto antedichos; en este caso, el que una persona o conjunto de personas sean objeto de una realización específica de CG es más bien casual (en el sentido de contingente). Dentro de la clase $B$ agrupo las menciones que se relacionan con una segunda posibilidad: que un acto de CG ocurra como reacción a una actuación o característica de un $\mathrm{O}$ particular (y no arbitrario); en este sentido, este $\mathrm{O}$ es necesario para la realización del acto.

\subsection{Escenario}

"Se refiere al tiempo y lugar de un acto de habla y, en general, a las circunstancias físicas". Para CG, cualquier escenario se considera, en general, posible. Las restricciones que mencionan algunos informantes tienen que ver más bien con la escena o con la cantidad de participantes: reuniones muy formales que tienen un orden establecido ("como una reunión de cancilleres"), "almuerzos", reuniones donde hay mucha gente. En todo caso, hay predilección por los lugares cerrados y un ambiente de privacidad. Por esto, se trata de evitar realizarlo, por ejemplo, en cines y en la iglesia. Parece más bien preferirse las "fiestas, reuniones de amigos y compañeros, entre familiares, grupos informales".

\subsection{Escena}

Consiste en el ambiente sicológico o cultural en que se desarrolla el acto de habla. Tomando como criterio el modo en que $\mathrm{O}$ se constituye en causante de la escena, se pueden dividir los tipos de disposiciones anímicas de los participantes en dos clases (vid. supra): (a) disposición hacia el entretenimiento o la diversión (v. fig. 6.1, clase A). (b) Diversos niveles de tensión o afección emocional por causa de la persona objeto de la conversación; se incluyen desde el simple deseo de expresar alguna idea sobre algún aspecto o actuación de $O$ hasta la desesperación por causa de una acción (sospechada, esperada o atestiguada) de su parte; por ser esta la clase de escena que más se especifica (v. fig.1, clase B), la considero como la principal ${ }^{8}$.

Figura 1

Escena

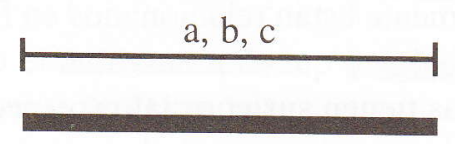

A: O casual

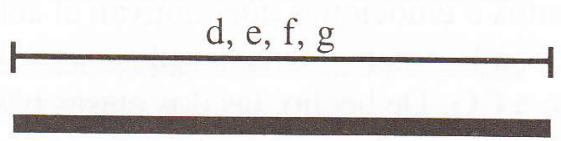

B: O necesario

A.

a: Ambiente de fiesta, alegría, deseo de diversión.

b: Aburrimiento, por tener algo de que hablar.

c: Deseo de burlarse [de alguien]; "por vacilar".

B.

d: Deseo de expresar alguna idea (sobre O); sorpresa.

e: Incomodidad, molestia; incertidumbre; temor; tristeza.

f: Resentimiento, envidia, malestar; cólera, tensión, enojo.

g: Desesperación, depresión, frustración, fracaso. 
Esas disposiciones anímicas no tienen que ser necesariamente compartidas por todos los participantes, pero conforman el ambiente sicológico en torno al acto. Sin embargo, "todos tienen que estar interesados en hablar [sobre O]".

\subsection{Participantes}

Como se dijo antes (vid. sección 6, supra), Hymes agrupa dentro de esta categoría cuatro componentes básicos: hablante, oyente, destinador y destinatario. Postula, además, que la categoría de participantes es, como tal, universal, y un aspecto que debe tomar en cuenta un estudio etnográfico serio. Sin embargo, al explicitar las reglas de habla para un evento (o un acto de habla) dado, es necesario especificar quiénes pueden o deben intervenir en ese evento particular. Con respecto a CG, los dos tipos de participantes que se mencionan para la conversación directa (la forma más general) son, como era esperable, los de hablante y oyente. Además, hay que apuntar que durante la dinámica de CG estos dos "roles" normalmente se intercambian.

En cuanto a la cantidad, el mínimo sería de 2, es decir, lo indispensable para el intercambio verbal; el máximo se concibe, en general, hacia los 7 u 8 participantes; además, la cantidad más común de participantes sería entre 3 y 4 . Sobre la edad, la tendencia es a considerar como mínima la adolescencia y como máxima la ancianidad; se excluyen, en todo caso, los extremos: niños menores de 6 años o "ancianos decrépitos". Respecto al sexo, por otro lado, el grupo puede estar conformado sólo por hombres, sólo por mujeres, o por hombres y mujeres.

\subsection{Propósitos convencionales}

En ellos está implícita la expectativa de un resultado o consecuencia convencional del acto (para los participantes en su conjunto). Para CG se aceptan propósitos muy variados, que van desde "pasar el rato" (entretenimiento), con lo cual no se busca explícitamente afectar la conducta de alguien hacia $\mathrm{O}$, hasta el extremo de querer excluir a $\mathrm{O}$ de un grupo o cortar a largo plazo la comunicación con él -recordemos que con "O" designamos el conjunto de personas objeto de CG, que puede ser una o varias-. Hay dos aspectos importantes sobre los propósitos del grupo como tal: (a) manifiestan deseos o intenciones que surgen de los sentimientos o emociones que motivan el acto. (b) Normalmente están relacionados en forma directa con la escena, considerada como el ambiente sicológico que domina durante o previamente a CG. De hecho, las dos clases básicas de escenas tienen sus correlatos respectivos en los propósitos: deseo de entretenimiento y necesidad de reaccionar verbalmente (en principio, aunque las consecuencias se pueden proyectar a otros tipos de conducta) ante la persona o personas a las que se considera responsables de cierto estado emocional. Sin embargo, dentro de la clase B (según fig. 2) se puede notar una subclase bien delimitada de propósitos. El criterio propuesto para la clasificación ha sido, nuevamente, el grado en que O se considera necesario o casual para lograr los fines del acto. Dentro de la clase B se utiliza el criterio adicional de la medida en que $\mathrm{O}$ se vería potencialmente afectado como consecuencia del acto: en $\mathrm{B}_{1}$ sería (en principio) cercana a cero, mientras que $\mathrm{B}_{2}$ incluye expectativas de efectos diversos en este sentido. 
Figura 2

Propósitos convencionales

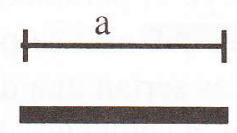

A: O casual

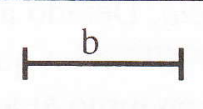

$\mathrm{c}, \mathrm{d}, \mathrm{e}, \mathrm{f}$

B: O necesario

$\mathrm{B}_{1}$

$\mathrm{B}_{2}$

A. a. Pasar el tiempo, "por vacilón". "Por tener algo para conversar".

$\mathrm{B}_{1}$. b. "Reafirmar lo que se opina [sobre O]: que a todos les cae mal".

$\mathrm{B}_{2}$. c. "Defender algún derecho" (ante terceros que puedan tener alguna influencia sobre la conducta de $\mathrm{O}$ ).

d. Cerrarle el paso en el grupo a O. Que se le pierda confianza en el grupo.

e. Hacer sentir mal a $\mathrm{O}$.

f. Desquitarse de O.

\subsection{Propósitos individuales}

Suponen metas o finalidades para cada participante. En este caso la situación es algo diferente: aunque hallamos de nuevo las dos clases básicas, el deseo de dañar de alguna forma a O (clase B, según fig. 3) es más radical; va desde "desenmascararlo", pasando por "crearle mal ambiente", hasta "despedazarlo". Con menor grado se mencionan propósitos considerados en algún sentido como constructivos (o al menos no destructivos): desahogo, "dar a conocer lo que es [O] para que los demás le tengan cuidado" o "que los demás no repitan lo malo [de O]". Dispuestos según los mismos criterios utilizados para los propósitos convencionales, los datos quedarían como en la figura 3.

Figura 3

Propósitos individuales

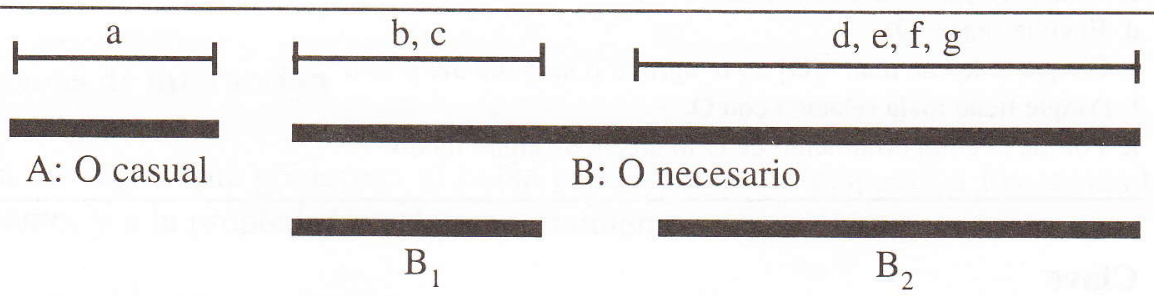

A. a. Pasar el rato. Ser parte del grupo.

$\mathrm{B}_{1}$. b. Desahogo; por sentirse mejor (respecto a las consecuencias de una acción de O sobre el participante)

c. "Para que los demás no repitan lo malo [de O]".

$\mathrm{B}_{2}$. d. "Desenmascararlo". "Divulgar los defectos" de O.

e. "Dar a conocer lo que es [O] para que los demás le tengan cuidado".

f. Dejar en mal a O. Ridiculizarlo. Desacreditarlo. "Crearle mal ambiente".

g. "Despedazarlo [a O]". 


\subsection{Motivos}

A veces, al preguntar por los propósitos se respondía más bien con motivos. Esto se puede justificar con base en una tendencia relativamente generalizada a confundir el por qué con el para qué o la causa con la consecuencia. Debido a esto, incluyo el parámetro motivos (razones, causas). Este se diferenciaría de la escena en que, mientras que esta se puede definir como la disposición sicológica inmediata en torno al acto, aquellos serían una disposición sicológica más bien mediata. En principio, los motivos determinarían la actuación de una persona hacia otra $(\mathrm{O}$, en nuestro caso) no sólo respecto a $\mathrm{CG}$ sino también respecto a otros actos verbales y no verbales. Por otro lado, motivos y propósitos se diferenciarían en la misma medida en que se diferencian causas y consecuencias (esperables).

Para CG, los motivos para convocar o ingresar al acto consisten, en general, en que uno o varios participantes se consideran (con la subjetividad del caso) afectados de algún modo y en algún grado por alguna actuación de O. Puede ser también sencillamente porque O no agrada a uno o a varios de los participantes. Se mencionan excepcionalmente: "vicio", costumbre, "por maldad", "complejo", sentimiento de frustración o fracaso; sin embargo, estos parecen relacionarse más bien con disposiciones usuales, permanentes o generales del participante potencial, que con una disconformidad específica hacia un O particular. También existe la sola búsqueda de un tema de conversación. En la figura 4, dispongo los datos según el criterio que he utilizado antes.

Figura 4

Motivos

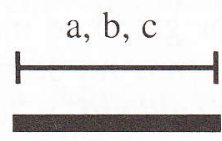

A: O casual

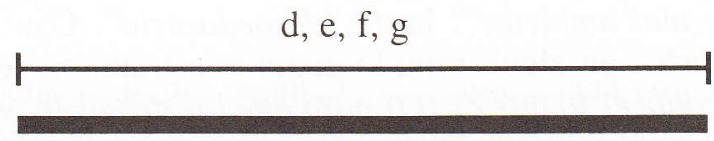

B: O necesario

A. a. Por tener algo de qué hablar.

b. Costumbre, "vicio"; por placer, por gusto; "por maldad".

c. "Complejo, frustración, fracaso".

B. d. Envidia (hacia O).

e. Porque $\mathrm{O}$ le cae mal. "[O] no le agrada a ninguno del grupo".

f. Porque tiene mala relación con $\mathrm{O}$.

g. Porque el comportamiento de $\mathrm{O}$ lo afecta de algún modo.

\subsection{Clave}

Es "el tono, manera o ánimo con que se desarrolla el acto". Puede depender (en orden de prioridad según el número de respuestas que lo mencionan): (a) de la disposición, mediata o inmediata, de uno o varios de los participantes, (b) del tema o contenido, (c) de la situación o del evento. Puede ir desde la jocosidad hasta la agresividad. Clasificados según si el o los participantes se consideran afectados objetivamente por $\mathrm{O}$, los datos podrían quedar como en la figura 5: 
Figura 5

Clave

\begin{tabular}{|l|}
\hline A: -afección \\
B. $\quad \begin{array}{l}\text { a. bromista, jocoso } \\
\text { b. burlón } \\
\text { c. enfático (por ej., porque "se cree tener razón sobre lo que se dice sobre él [O]") } \\
\text { d. serio } \\
\text { e. tenso (por reservas sobre la opinión de los otros participantes) } \\
\text { f. de enojo, agresivo, alterado }\end{array}$ \\
\hline
\end{tabular}

\subsection{Canal}

Son los medios de transmisión del habla. Excepto para una entrevistada ("oral siempre", incluso se excluye el teléfono), todos los demás coinciden en que no hay restricción alguna; puede ser oral, escrito, electrónico siemplos: "papelillos", carta, internet, teléfono, dibujos-. No obstante, la forma menos marcada sería la oral, sobre todo la directa9 .

\subsection{Registro}

Los registros son los distintos grados de formalidad en la forma de hablar, escogidos según las circunstancias o la situación de habla. Las opiniones más comúnes son, según el número de menciones, que para CG el registro: (a) depende de la situación o del evento (4 menciones); (b) depende de aspectos de los participantes (4 menciones), específicamente, de la jerarquía entre ellos (ejemplos: entre compañeros, jefe-subalterno(s), profesor-alumno(s), sacerdote-feligrés(es)) o de la clase social; (c) siempre es al menos tan neutro o informal como el habla cotidiana (3 menciones).

\subsection{Normas de interacción}

Son las reglas que gobiernan el habla específicamente respecto a los requisitos para los participantes y a la propiedad en el comportamiento.

\subsubsection{Sobre los participantes}

(a) Se considera como lo más relevante el que haya un relativo nivel de confianza entre ellos. En todo caso, la confianza debe ser mayor o al menos igual entre los participantes que la que hay entre estos y O. También se requieren las siguientes condiciones: (b) solo quienes conozcan a $\mathrm{O}$ son participantes potenciales, (c) "todos tienen que estar interesados en hablar 
[sobre aquel]" (mencionado ya en 6.2: escena) y (d) quien es objeto de la conversación no puede intervenir en esta.

\subsubsection{Sobre las normas de comportamiento}

Las normas de comportamiento para CG tienen que ver, en general, con convenciones establecidas y normalmente sobreentendidas dentro del grupo de participantes. Hay 5 menciones sobre la existencia de normas dependientes de la situación: la propiedad en el comportamiento puede estar determinada por aspectos diversos de la situación, especialmente por el momento o lugar. Por ejemplo, en lo tocante a la altura de la voz, normalmente se hablará en voz baja, aunque, si en la situación o el evento predomina la conversación en voz alta, el acto puede tomar esta característica (lo importante es que quede resguardada la privacidad necesaria; recordemos 6.1, escenario). Sin embargo, hay también reglas independientes de la situación; en la figura 6 incluyo los tipos más representativos de contravenciones a estas últimas, de las cuales se deducen las normas respectivas:

Figura 6

Conductas reprochables independientes de la situación

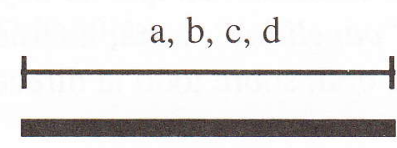

A: -reprochable

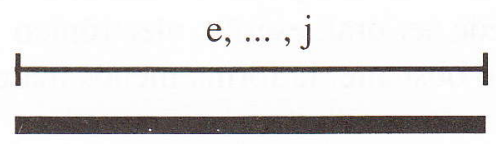

B: +reprochable

A. a. Interrumpir al hablante de turno.

b. Que el comentario sea muy extenso.

c. Denigrar a O con vocabulario demasiado grotesco.

d. Querer salir de la conversación sin haber terminado.

B. $\quad$ e. "Que alguien rectifique a lo último"; desdecirse.

f. Reprochar la conversación.

g. Titubear, mostrar inseguridad.

h. Monólogo ("acaparar la conversación").

i. Silencio absoluto o demasiado prolongado (aunque sí puede haber silencio participativo).

j. "Criticarse entre sî".

Las penalizaciones pueden ser inmediatas, a mediano o a largo plazo, verbales o gestuales, pasajeras o permanentes, leves o drásticas. Dependen de la gravedad del comportamiento considerado negativo. Como ilustración de las formas de castigo inmediatas se pueden señalar las correspondientes a las infracciones (f), (h), e (i), que consisten en "salirse" del acto, darlo por terminado o excluir de él al infractor. Como reacción a (j), el o los atacados "contraatacan" al que hizo el comentario. En otro nivel, (f), y tal vez otras de las conductas de la clase B, pueden provocar que quien las manifiesta no sea aceptado nuevamente para CG por este grupo. 


\subsection{Normas de interpretación}

"Implican el sistema de creencias de una comunidad" en la medida en que determinan cómo interpretar un acontecimiento dado.

Hay coincidencia general en concebir el acto de CG como negativo en algún sentido (e incluso "cien por ciento negativo"), ya sea para los participantes o por las consecuencias (esperables o posibles) para O. Manifiesta antivalores culturales (odio, deseo de destrucción, etc.) o al menos estados emocionales no deseables para los participantes (cólera, tensión, resentimiento, etc.). Se considera tanto como un medio para perjudicar de alguna manera a alguna persona como un acto que por sí mismo puede dañar la imagen del que lo realiza. Aun en el caso en que se convoca o se inicia por entretenimiento, la característica dominante es cierta malicia en él.

\subsection{Género}

Son "categorías tales como poema, mito, cuento, proverbio, (...), oración, editorial, etc.". La opinión predominante es que para CG es siempre conversacional ${ }^{10}$.

\subsection{Contenido}

Debido a la subjetividad de la persnectiva con que se tratan los posibles contenidos de $\mathrm{CG}$, prácticamente no existen restricciones sobre los temas. Hay que anotar, sin embargo, que el objeto es siempre y necesariamente una persona (o varias) ${ }^{11}$. Casi cualquier comportamiento, característica (física, sicológica, intelectual, etc.), actitud o costumbre de la persona que es constituida en objeto del acto serían válidos; incluso las circunstancias que fortuita o involuntariamente lo afectan, como que $\mathrm{O}$ fuera ascendido de su puesto o que ganara la lotería. Tan es así que aun características físicas naturales pueden servir para tal efecto. Se incluyen aspectos familiares, económicos, emocionales, sexuales, profesionales, relaciones personales, forma de vestir, defectos de personalidad, cosas que hace o que no hace, etc. En síntesis, "cualquier cosa".

\subsection{Forma}

Hymes no define explícitamente en qué consiste, pero se relaciona con los medios de expresión y las formas de hablar compartidas. Para el análisis de este aspecto, diferenciaremos entre convocatoria, cuerpo y finalización del acto.

\subsubsection{Convocatoria}

Entiendo por convocatoria, siguiendo a Schegloff (1986), la secuencia de apertura de una conversación. En nuestro caso, generalmente es espontánea, aunque puede ser planeada. Además, puede ser verbal o no verbal (o mixta); si es verbal puede ser directa o indirecta, explícita o no. Incluye gestos, silbidos, frases, movimientos o contactos corporales, miradas... La característica predominante es, de nuevo, la sobreentendida malicia del llamado, cuyas diver- 
sas manifestaciones se interpretan convencionalmente entre los participantes como invitación a CG. Como ejemplos representativos de las formas posibles tenemos:

\section{Cuadro 1}

Formas de convocatoria

\begin{tabular}{|c|c|}
\hline No verbal & $\begin{array}{l}\text { - acercamientos corporales } \\
\text { - golpes suaves en brazos o espalda } \\
\text { - señales de llamado con las manos } \\
\text { - gestos en general } \\
\text { - ruidos, silbidos } \\
\text { - miradas de secreto, suspicacia o malicia }\end{array}$ \\
\hline $\begin{array}{l}\text { Verbal directa } \\
\text { (supone suficiente } \\
\text { cercanía local o } \\
\text { temporal de O y se } \\
\text { confunde con el } \\
\text { cuerpo del acto en sí) }\end{array}$ & $\begin{array}{l}\text { "Mirá cómo anda de pintada." } \\
\text { "QQuién iba a decir..." } \\
\text { "Tan santico que se ve!" } \\
\text { "Puramente calle doce..." } \\
\text { "YYa me tiene...[seguido de gesto]!" } \\
\text { "No sirve para nada...!" } \\
\text { "Ese mae es un cochino..." } \\
\text { "QQué casa más sucia!"12 }\end{array}$ \\
\hline Verbal indirecta & $\begin{array}{l}\text { "Te acordás de fulano de tal..." } \\
\text { ¿Sabe a quién vi en el parqueo...?" }\end{array}$ \\
\hline Verbal explícita & "Vení, comamos gente." \\
\hline Verbal no explícita & $\begin{array}{l}\text { "Vení, veni...!" } \\
\text { "Venga para contarle una cosa." }\end{array}$ \\
\hline
\end{tabular}

\subsubsection{Cuerpo del acto}

La altura de la voz depende de la situación o del evento (vid. 6.10.2, supra): aunque lo normal es que se hable en voz baja o hasta en murmullo, en fiestas o reuniones muy informales se puede obviar esta condición. Aparte de esto, no hay un orden o forma establecidos y se desarrolla como una conversación común.

\subsubsection{Finalización}

Puede ser absoluta o relativa: absoluta si con eso se da por concluido el acto de CG para todos los participantes, relativa si consiste en la retirada del acto de solo algún(os) participante(s). Puede ser voluntaria o forzada: si es voluntaria, puede ser por "que se acabó el tema" o "por cansancio"; si es forzada, puede ser porque es tiempo de dedicarse a otras labores o por la llegada de alguna persona que no se desea incluir como participante. Por otro lado, puede ser verbal (directa o indirecta, explícita o no explícita) pero también no verbal (normalmente simultánea con o precedida por una forma verbal, aunque en ciertas ocasiones esta se 
puede obviar). En el cuadro 2 se ejemplifican las diversas manifestaciones. Incluyo el silencio como una forma verbal indirecta.

\section{Cuadro 2}

Formas de finalización

\begin{tabular}{|c|l|}
\hline No verbal & $\begin{array}{l}\text { Uno, varios o todos los participantes (dependiendo } \\
\text { de si es relativa o absoluta) se comienzan a disgregar. } \\
\text { Dedicarse a hacer otra cosa. } \\
\text { Levantarse si estaban sentados o viceversa. }\end{array}$ \\
\hline Verbal directa & $\begin{array}{l}\text { "Bueno, que no salga del barrio [broma]." } \\
\text { "Diay, ¿qué se puede hacer..?" } \\
\text { "Bueno, ya me tengo que ir" } \\
\text { "Ay sí, qué feo estar uno en eso, ¿verdad?" }\end{array}$ \\
\hline Verbal indirecta & $\begin{array}{l}\text { Se cambia el tema de conversación o todos los } \\
\text { participantes dejan de hablar. }\end{array}$ \\
\hline Verbal explícita & "iBueno ya, dejemos de comer gente!" \\
\hline $\begin{array}{l}\text { Verbal no } \\
\text { explícita }\end{array}$ & "Pero ya, dejemos esta vara por hoy!" \\
\hline
\end{tabular}

\section{Interpretación y conclusiones}

Un acto de CG típicamente caracterizado se daría bajo las siguientes condiciones (las alternativas en escena, propósitos, motivos y clave parecen corresponderse respectivamente):

\section{Cuadro 3}

\section{Componentes de una realización típica de CG}

\begin{tabular}{|l|l|}
\hline escenario & $\begin{array}{l}\text { cualquiera con suficiente privacidad. } \\
\text { ambiente de diversión o afección emocional de uno, varios o todos los } \\
\text { participantes hacia O. }\end{array}$ \\
tres; cualquier edad entre la adolescencia y la ancianidad; cualquier sexo; \\
participantes \\
propósitos \\
divertirse o dañar la imagen de O. \\
tener un tema de conversación o sentirse afectado por la actuación de la \\
persona objeto. \\
jocosa o de enojo. \\
clave \\
canal directo. \\
registro \\
normas de interacción de la situación, del evento o de las calidades de los participantes. \\
los participantes deben tenerse confianza entre sí; la persona objeto no puede \\
estar presente; todos deben conocer a O y tener interés en hablar sobre él; no \\
desdecirse, no mostrar inseguridad, no criticarse entre sí, no permanecer de- \\
masiado tiempo en silencio... \\
es un acto negativo, dañino. \\
conversacional. \\
cualquier tema sobre otra persona. \\
se convoca espontáneamente con cualquier forma de llamado; se habla en voz \\
conero \\
contenido; se finaliza espontáneamente o con alguna señal verbal o corporal. \\
forma
\end{tabular}


Comenzaré por una acotación sobre la denominación cultural del acto en su enunciación genérica ${ }^{13}$. El primero de los dos términos hace referencia a la forma natural de tomar alimento, que estriba en llevarse algo a la boca, morderlo, masticarlo y tragarlo. Al comer se desgarra el alimento, se destroza. Esto es también lo que ocurre, a un nivel simbólico, con aquella persona de la que se ocupan los participantes en CG. Como se vio en 6.4 y 6.5, la intención de perjudicar que se manifiesta en la clase B de ambos tipos de propósitos (figs. 2 y 3, respectivamente) se muestra por sí misma. Por otro lado, he mencionado ya la mayor radicalidad de la reacción hacia $\mathrm{O}$ que hay en los propósitos individuales, entre los que se hallan algunos tan crudamente expresados como "desenmascararlo", "ridiculizar [1o]", e incluso "despedazarlo". Esto último es justificación suficiente de por qué se liga este acto con el de comer. Por otro lado, al comer se satisface una necesidad natural, lo que puede implicar incluso placer (si se tenía mucha hambre o si aquello es muy delicioso). Del mismo modo, CG llena una necesidad: de ocupar el tiempo, de diversión; de desahogo, defensa o venganza (v. 6.4 y 6.5). Con esto se entiende por qué se puede realizar también como entretenimiento y a veces con gran gusto.

El segundo término de la expresión que nos ocupa encuentra su justificación en 6.13 (contenido): el objeto del acto es siempre algún aspecto de una persona. En efecto, el rasgo [+humano] provee la delimitación básica del universo de objetos, que tiene un reflejo linguiístico a priori en el nombre mismo de CG -otra restricción es impuesta por la dinámica social ligada al nombre: la persona de la cual se trata en una realización particular de CG no debe estar presente cuando "se la están comiendo"-. Como he mencionado, se puede tratar de una o de varias personas, a lo que puede estar haciendo referencia el término gente. Sin embargo, hay otra razón posible, que no trataré de validar aquí aunque es de interés mencionar, y es que no aluda a un referente colectivo necesariamente por su simultaneidad sino por su acumulación sucesiva en la memoria grupal o colectiva (en el mismo sentido en que un médico puede decir hoy voy a atender mucha gente, con lo cual no quiere afirmar necesariamente que los verá a todos al mismo tiempo).

Hay varios puntos interesantes que, por razones de extensión, sólo puedo dejar brevemente señalados. Uno de ellos es la connotación tabuesca de CG, pues CG motiva asociaciones con otra expresión de idéntica forma pero de sentido literal: comer gente como acto de canibalismo. La privacidad buscada para CG (ver 6.1: escenario) tiene el carácter de lo prohibido más que de lo íntimo, cuando se une con la interpretación socialmente negativa de nuestro acto (según 6.11). En efecto, cuando aquello que comemos es también humano, estamos ante un acto de antropofagia, considerado entre lo más bajo que se pueda hacer, entre lo socialmente más reprochable. De ahí el matiz siempre negativo que tiene comer gente, síntesis de la paradoja entre algo no permitido pero en alguna medida necesario.

Segundo, CG no consiste solamente en un informe o descripción; aunque existan pretensiones de objetividad, estas son directamente influenciadas por los motivos (6.6), lo que a la vez se evidencia en la clave (6.7). Esta ofrece dos posibilidades básicas típicamente realizadas: la jocosidad o el enojo, las cuales difícilmente permitirían o surgirían de la objetividad.

En tercer lugar, lo siguiente: CG refleja diversas actitudes propias de la pertenencia a un grupo relativamente cerrado, aspecto manifiesto en varios de sus componentes. Por un lado, la posibilidad de integrarse o ser integrado en la conversación está de previo condicionada por la confianza, el interés, el conocer a la persona de quien se habla y, lo que es más sintomático, la necesidad de no incluir en el acto a quien es objeto de la conversación (esto fue tratado en 6.10: normas de interacción, sobre los participantes). Por otro lado, en la figura 6.6 
hallamos dos clases claramente diferenciadas de normas de comportamiento. Las de la clase A parecen ser normas más generales de interacción verbal en nuestra cultura; las de la clase $\mathrm{B}$, en cambio, tienen que ver con concepciones sobre la pertenencia a un grupo cerrado ${ }^{14}$, y precisamente a estas últimas corresponden las penalizaciones más extremas. Es interesante resaltar como ejemplo, la significación del silencio total o demasiado prolongado de un participante, el cual implica valores negativos como deslealtad al grupo, disconformidad con la opinión reinante en el mismo, miedo, hipocresía, cobardía, posible traición, etc. El silencio prolongado de una parte provoca tensión en las demás, y puede ser señal, según un informante, de que "es muy amigo de él [O] y después corre a contarle todo". Pero recordemos que el silencio de todos los participantes sería más bien una señal aceptable y convencional para dar por concluido el acto como tal (v. cuadro 6.2). En fin, también en las formas de convocatoria y de finalización encontramos una serie de opciones cinésicas cuya apropiada descodificación requiere compartir elementos semánticos que permitan comprenderlas como tales ${ }^{15}$.

Para finalizar, queda también propuesto para análisis futuros el tema de la estructuración y jerarquización de los componentes de CG: al parecer, los motivos constituyen el generador del acto, proyectándose en las clases respectivas de propósitos, escenas y claves; el canal oral directo y el género conversacional, aunque ligados entre sí, serían independientes de los demás factores; los escenarios y algunos aspectos de la convocatoria y de la finalización, estarían justificados por la valoración negativa según la cual se interpreta el acto; esta, a su vez, tendría su base en los contenidos, debido a la censura social hacia el daño intencional a otros seres humanos, aun a nivel simbólico. Quedan también demostradas las drásticas consecuencias que podría tener para una persona el ser objeto de este tipo de conversación, es decir, el poder social de la misma; así también para quien la practica habitualmente.

\section{Notas}

* Deseo agradecer a las personas que tan amablemente accedieron a la entrevista; asimismo, a Carla Jara por la lectura de los borradores y por las múltiples sugerencias que me ofreciera y a Guiselle Vargas por la lectura del último borrador y las correcciones finales. Por supuesto, todos los errores que subsistan son de mi responsabilidad.

1. Gran parte del artículo de Dickeman (1982) se dirige a justificar por qué es en el terreno del lexicón donde será más fructífero este tipo de indagaciones sobre la relación lengua-cultura. Una de las principales razones es la dificultad de definir con pertinencia los rasgos culturales y los rasgos lingüísticos cuya correlación se va a analizar, siendo que los primeros deben ser propiamente ofrecidos por la antropología cultural, mientras que los segundos por la linguística y, además, en forma independiente. Un intento de relacionar el "orden básico SOV" con una "tecnología de alto nivel", por ejemplo, es poco plausible, mientras que se ha encontrado una fuerte relación implicacional (no necesariamente causal) entre la complejidad de los sistemas de colores y el grado de avance tecnológico de un grupo cultural.

2. Rona (1974: 210) la delimita así: "El término de sociolingüística se aplica más bien a los estudios de la variación lingüística dentro de una misma sociedad, y nosotros entendemos que debe continuar aplicándose asî". Por su parte, Dickeman (op. cit.: 118-9) afirma, sobre esta disciplina, que: "Dichos estudios describen relaciones dentro de una lengua particular, en donde algún rasgo específico o algún conjunto de rasgos sirve para indicar diferencias socialmente significativas entre los hablantes, como decir, de clases, o de edad". 
3. En realidad, la diferencia principal entre la etnografía antropológica y la etnografía del habla, disciplina socio o etnolingüística, consiste en que la primera intenta una descripción exhaustiva de los diferentes aspectos de la interacción socialmente normada dentro de una comunidad diferenciada culturalmente. En otras palabras, no se concentra, como sí la segunda, en las actividades cuyo eje es el habla. Esto no significa, sin embargo, que la primera desconozca el papel determinante del lenguaje para la permanencia y transmisión de los sistemas socialmente constituidos. "Aun las etnografías que nosotros tenemos, aunque casi nunca enfocadas en el habla, nos muestran que las comunidades difieren significativamente en las formas de habla, en los patrones de repertorio y switching, en los roles y significados del habla. Nos señalan diferencias con respecto a las creencias, valores, grupos de referencia, normas y demás, cuando estos entran en el progresivo sistema del uso del lenguaje y su adquisición por los niños" (Hymes 1986: 42).

4. La Región Central "comprende los amplios valles en el interior del país con sus zonas montañosas colindantes" (Carvajal 1995: 62), estando el grueso de su población aglutinada en los cuatro grandes centros urbanos de Cartago, Alajuela, San José y Heredia. Se caracteriza, para mencionar dos de los aspectos más importantes, (1) "por ser la única con crecimiento económico autosostenido, en ella se encuentran las funciones urbanas más preeminentes: comerciales, financieras, industriales, administrativas, gracias a las cuales goza de un rol de primer orden en la vida económica del país" (ibid: 64) y (2) porque, aunque ocupa solo el 15\% de la superficie del país, para el Censo de 1984 incluía el 64.4\% de la población total, con tendencia hacia una mayor concentración. Dentro de esta región, es de particular importancia la Aglomeración Metropolitana de San José, que presenta la mayor concentración humana del país, así como el principal conjunto urbano-residencial-industrial.

Aunque estos factores no expresan directamente una relevancia como unificadores culturales ni, en particular, lingüísticos de algún tipo, en el concepto mismo de región hay implícita una homogeneidad en la interrelación humana, en la cohesión de sus habitantes y en la percepción del espacio vivido (cfr. Carvajal op. cit.: 45). En síntesis, la conformación geográfica (en sentido amplio, no sólo físico) de la Región Central permite postularla como un sistema interaccional relativamente unitario.

5. Dentro del nivel de análisis de este trabajo me interesa una caracterización global de CG, por lo que no entraré a considerar variables como sexo, ocupación, etc. más que para especificar, en términos generales, al grupo de informantes.

6. Hymes (1986) los llama, respectivamente, propósitos-resultados (purposes-outcomes) y propósitos-metas (purposes-goals).

7. Nuevamente, en los casos en que Hymes (1986) ofrece una definición del componente, la transcribo directamente. Cuando no, trato de construir una a partir de sus explicaciones, a menos de que sea una noción de uso generalizado en lingüística.

8. Para el presente trabajo me parece suficiente, en este y en los demás componentes, la agrupación en clases. Por lo tanto, aunque implícitamente he tratado de ordenar los datos dentro de cada clase. no propongo aquí criterios específicos para tal efecto.

9. Según cuatro informantes, se incluyen "programas para el pueblo", interactivos o no (programas radiofónicos o televisivos, de variedades musicales o deportivos); otra respuesta incluye el periódico. Sin embargo, es mi opinión que los canales de este tipo carecen de la relativa privacidad que sería requisito para CG (véase sección 6.1), por lo que la alusión a los mismos, según considero, se debe a la ya mencionada intersección con otros actos.

10. Un informante incluye el editorial (de un periódico); otro, la mesa redonda. No obstante, por razones similares a las ofrecidas para excluir ciertos canales aun cuando fueran citados por algunos entrevistados (ver nota anterior), considero que estos se deben suprimir aquí. 
11. Existen formas de comer gente en que el objeto humano puede estar implícito. Como muestra, tomemos la expresión "iqué casa más sucia!”: bajo las condiciones (léase componentes) adecuadas, puede interpretarse como parte o incluso la totalidad de un acto de CG. Esto quiere decir que el objeto no sería la casa, sino una persona hacia la cual la referencia se da por asumida.

12. Ver nota 11 .

13. Aunque me he venido refiriendo a CG por el nombre genérico de comer gente, esta es más bien su forma menos visual; en efecto, las realizaciones específicas particularizan el objeto en formas como: "me estaban comiendo", "se estaban comiendo a Juan", "hasta entre ellos mismos se comen", etc.

14. No puedo extenderme aquí sobre las motivaciones que provocan que una conducta determinada sea interpretada como violación a una norma implícita, aun cuando muchas veces los entrevistados aportaron esa información.

15. Cfr. La Barre 1978 .

\section{Bibliografía}

Albert, Ethel M. 1986. "Culture Patterning of Speech Behavior in Burundi”. En: Gumperz y Hymes (eds.), 72-105.

Carvajal Alvarado, Guillermo. 1995. Geografia, Espacio y Regiones en Costa Rica. San José: Guayacán.

Dickeman Datz, Margaret. 1982. "Hacia la búsqueda de una relación entre lenguaje y cultura: Reflexiones de una lingüista”. Revista de Filología y Lingüística. 8 (1-2): 113-20.

Fishman, Joshua A. 1966/1977. "Una sistematización de la hipótesis whorfiana". En: Smith (comp.), 165-81.

Frake, Charles. 1974. "Cómo pedir una bebida en Subanun”. En: Garvin y Lastra (comps.), 117-24.

Garvin, Paul L. y Yolanda Lastra de Suárez (comps.). 1974. Antología de Estudios de Etnolingüística y Sociolingüística. México D.F.: Universidad Nacional Autónoma de México.

Gumperz, J. 1962/1974. "Tipos de comunidades lingüísticas”. En: Garvin y Lastra (comps.), 234-46.

Gumperz, J. y D. Hymes (eds.). 1986. Directions in Sociolinguistics: The Ethnography of Communication. Oxford: Basil Blackwell.

Hymes, Dell. 1964/1974. "Hacia Etnografías de la Comunicación”. En: Garvin y Lastra (comps.), 48-89. 
1986. "Models of the Interaction of Language and Social Life". En: Gumperz y Hymes (eds.), 35-71.

Hymes, Dell (comp.). 1964. Language in Culture and Society: A Reader in Linguistics and Anthropology. New York: Harper \& Row, Publishers.

Jara, Carla V. 1986. "Recopilación y análisis de juegos de palmadas". Revista de Filología y Lingüística. 12(1): 103-21.

La Barre, Weston. 1978. "Paralinguiística, cinésica y antropología cultural”. En: Sebeok et al., 251-89.

Muñoz Barquero, Elizabeth. 1978. Lenguaje y Realidad en Adam Schaff. Tesis de Licenciatura: Universidad de Costa Rica.

Nadel, S.F. 1964. "Morality and Language among the Nupe". En: Hymes (comp.), 264-6.

Rona, José Pedro. 1970/1974. "La concepción estructural de la sociolingüística". En: Garvin y Lastra (comps.), 203-16.

Rossi-Landi, Ferruccio. 1974. Ideologías de la Relatividad Lingüística. Buenos Aires: Ediciones Nueva Visión.

Schegloff, Emanuel A. 1986. "Sequencing in Conversational Openings". En: Gumperz y Hymes (comps.), 346-80.

Sebeok, Thomas A. et al. 1978. Semiótica Aplicada. Buenos Aires: Ediciones Nueva Visión.

Smith, Alfred G. (comp.). 1977. Comunicación y Cultura (3): Semántica y Pragmática. Buenos Aires: Ediciones Nueva Visión.

Umaña Aguilar, Jeanina. 1988. "En torno a los métodos de la sociolingüística”. Revista de Filología y Lingüística. 14(1): 83-94.

Wardhaugh, Ronald. 1992. An Introduction to Sociolinguistics. 2a. ed. Oxford: Basil Blackwell.

Wierzbicka, Anna. 1992. Semantics, Culture and Cognition: Universal Human Concepts in Culture-Specific Configurations. New York/Oxford: Oxford University Press. 